phys. stat. sol. (b) 206, 153 (1998)

Subject classification: 78.66.Fd; 78.55.Cr; S7.12; S13

\title{
Mapping of the Local Confinement Potential in Semiconductor Nanostructures by Near-Field Optical Spectroscopy
}

Ch. Lienau ${ }^{1)}$ (a), A. Richter (a), G. Behme (a), M. Süptitz (a) T. Elsaesser (a), M. Ramsteiner (b), R. Nötzel (b), and K.H. Ploog (b)

(a) Max-Born-Institut für Nichtlineare Optik und Kurzzeitspektroskopie, D-12489 Berlin, Germany

(b) Paul-Drude-Institut für Festkörperelektronik, D-10117 Berlin, Germany

(Received September 1, 1997)

The optical properties of a new quantum-well-embedded GaAs quantum wire structure grown on patterned (311)A GaAs surfaces are directly mapped by low temperature near-field scanning optical microscopy (NSOM). Photoluminescence excitation spectroscopy with subwavelength spatial resolution allows a quantitative analysis of the local confinement potential of such structures which exhibit shallow barriers close to the quantum wire. This local potential influences the carrier transport dynamics within the embedding quantum well and - in particular - carrier trapping from twodimensional quantum well into one-dimensional wire states as is directly revealed in steady-state and time-resolved photoluminescence experiments at variable temperatures.

\section{Introduction}

Spatial variations of the bandstructure on a nanometer scale by a controlled change of the material composition are the basis of semiconductor nanostructures in which a lowdimensional confinement of carriers is realized. In such systems, both the optical spectra and the dynamics of elementary excitations are distinctly different from spatially homogeneous (bulk) materials. The study of such properties requires a characterization of the local confinement potentials. Until now, techniques based on the injection of electrons such as cathodoluminescence spectroscopy [1,2] or ballistic electron emission microscopy $[3,4]$ have mainly been used for monitoring the local bandstructure of subsurface nanostructures with high spatial resolution. However, the initial nonequilibrium energy distribution of carriers and its relaxation towards quasi-equilibrium, both affecting the experimental signals, are difficult to control in such measurements. In contrast, all-optical spectroscopy allows for resonant excitation and - thus - generation of carriers under well-defined conditions. To make use of these advantages, it is necessary to overcome the diffraction limit, e.g. by using near-field optical techniques with subwavelength spatial resolution $[5,6]$.

In this paper, we report new experimental results combining low-temperature nearfield optical microscopy and photoluminescence excitation (PLE) spectroscopy to directly map the local confinement potential of a novel GaAs quantum wire structure

$\left.{ }^{1}\right)$ Fax: ++49 306392 1476; e-mail: lienau@mbi-berlin.de 
grown on patterned (311)A GaAs surfaces. Local energetic barriers separating quantum wire (QWR) and embedding quantum well (QW) are identified and their effect on the trapping of carriers from two-dimensional QW into one-dimensional QWR states is demonstrated in near-field photoluminescence experiments at variable temperatures. The real-space motion of carriers is monitored in time and space by combining subwavelength spatial and picosecond temporal resolution.

\section{Experimental}

The QWR structure investigated in our experiments is depicted schematically in Fig. 1a. The sample was grown by molecular beam epitaxy on patterned GaAs (311)A substrates at the sidewall of 15 to $20 \mathrm{~nm}$ high mesa stripes oriented along [01-1] [7]. The sample consists of a nominally $6 \mathrm{~nm}$ thick GaAs QW layer clad by $50 \mathrm{~nm}$ thick $\mathrm{Al}_{0.5} \mathrm{Ga}_{0.5} \mathrm{As}$ barriers. Formation of a sidewall QWR arises from the preferential migration of Ga atoms within the QW layer from both the mesa top and the mesa bottom towards the sidewall [8]. Cross-sectional TEM images [7] indicate that - as a consequence of this migration process - the thickness of the GaAs QW near the sidewall

(a)

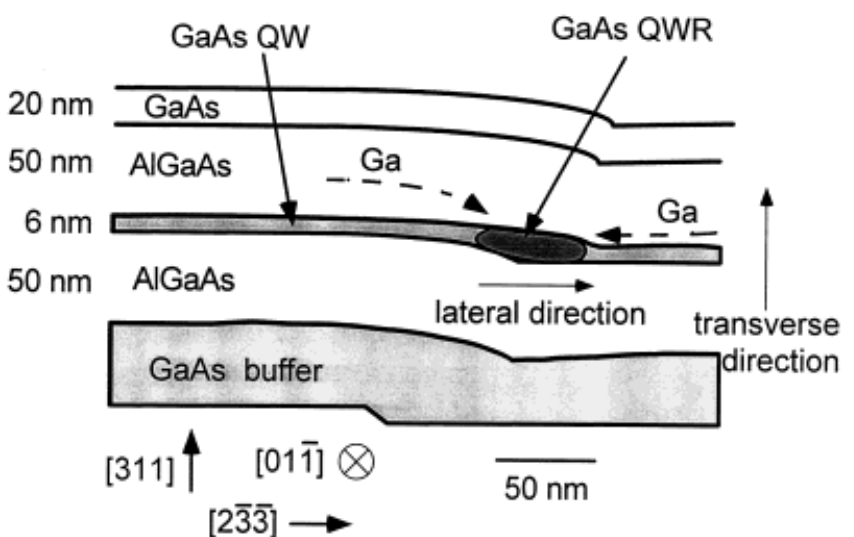

(b)

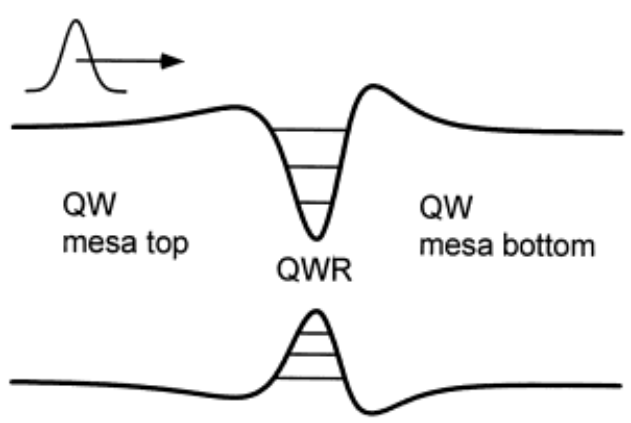

Fig. 1. a) Schematic of the quantum wire structure. The one-dimensional confinement originates

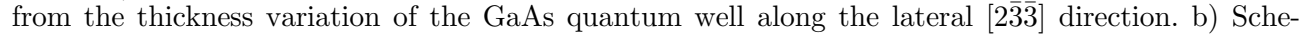
matic energy diagram: potential energy and subband structure along the lateral direction. Diffusion of carriers after localized excitation in the quantum well is depicted schematically 
increases from 6 up to $13 \mathrm{~nm}$. This change in QW thickness results in a $1 \mathrm{D}$ confinement over a lateral QWR width of $50 \mathrm{~nm}$ (see Fig. 1b). Confinement of excitons in the wires was independently inferred from the diamagnetic shift of the QWR photoluminescence (PL) [9]. For device applications it is important that these wires exhibit high uniformity, narrow PL emission lines and high PL efficiency up to room temperature. They can be vertically stacked in the growth direction without increase in interface roughness and wire size fluctuations.

Time- and spatially resolved near-field spectroscopic experiments on this QWR sample at temperatures between 10 and $300 \mathrm{~K}$ are performed with a newly developed near-field scanning optical microscope (NSOM) [10]. In this instrument, the sample is cooled by attaching it to the cold finger of a continuous flow liquid helium cryostat, the temperature of which can be varied between 8 and $330 \mathrm{~K}$. Cold finger, sample, and the NSOM scan head, consisting of a near-field fiber probe [11], a tuning fork [12], shear-force distance regulation $[13,14]$, and coarse and fine positioning, are mounted inside a vacuum chamber. In this way, the sample can be cooled to low temperatures while leaving the temperature of the NSOM scan head at room temperature. Probe tips were made by pulling single-mode optical fibers to a sharp taper in a commercial fiber puller and then coating the taper with a 50 to $100 \mathrm{~nm}$ thick aluminum or gold layer. The diameter of the aperture at the end of these probes can be controlled between less than 100 and $200 \mathrm{~nm}[15,16]$.

The experiments reported in this paper were performed in an illumination geometry [17]. Spatial resolution is provided in the excitation process by transmitting light through the probe tip and photoluminescence emitted by the sample is collected in the far-field by a conventional microscope objective. The luminescence is dispersed in a $0.25 \mathrm{~m}$ double monochromator with a spectral resolution of $1.2 \mathrm{~nm}$ and detected with a silicon avalanche photodiode either in steady state or with a time resolution of $250 \mathrm{ps}$. Different tunable lasers served for steady state or time-resolved excitation of the sample with powers between 10 and $100 \mathrm{nW}$, corresponding to a very low carrier density between $10^{4}$ and $10^{5} \mathrm{~cm}^{-1}$.

\section{Near-Field Excitation Spectroscopy: Mapping of the Local Bandgap}

In a first series of measurements, the subwavelength spatial resolution of NSOM was combined with PLE spectroscopy for mapping the confinement potential of the QWR sample. At a sample temperature of $8 \mathrm{~K}$, the QWR emission appears as a single narrow peak centered at $1.545 \mathrm{eV}$ with a spectral full-width-at-half-maximum (FWHM) of $8 \mathrm{meV}$ (Fig. 2b). QW emission is centered around $1.604 \mathrm{eV}$ with a spectral width of $12 \mathrm{meV}$. Information on the local energetics of the QWR and the embedding QW structure is obtained from spatially resolved PLE spectra. In such an experiment, the sample is locally excited through the NSOM probe and the tip is scanned along the $y$ direction perpendicular to the QWR located at $y=0$. In Fig. $2 \mathrm{a}$, the intensity of QWR luminescence at a detection energy of $1.544 \mathrm{eV}$, i.e. slightly below the maximum of QWR emission, is plotted as a function of excitation energy (abscissa) and of lateral distance of the exciting fiber tip from the QWR position at $y=0$ (ordinate). We find a PLE spectrum that is spatially confined to the QWR region. For excitation energies below $1.63 \mathrm{eV}$, the width of this region has a value of about $300 \mathrm{~nm}$ and is limited by the finite distance of 
(a)

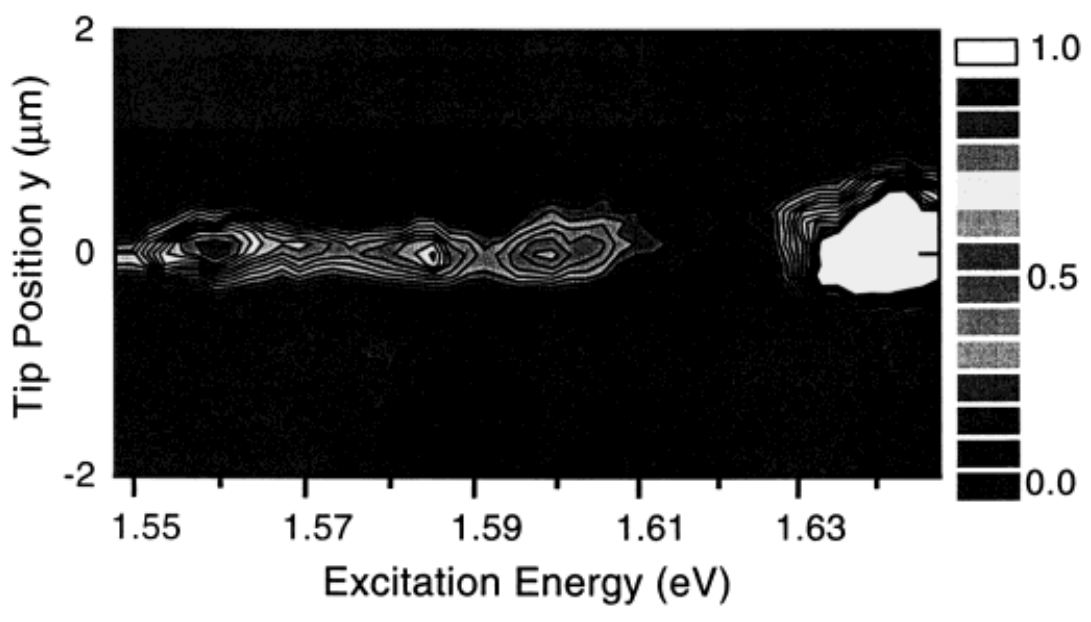

(b)

Excitation Energy (eV)

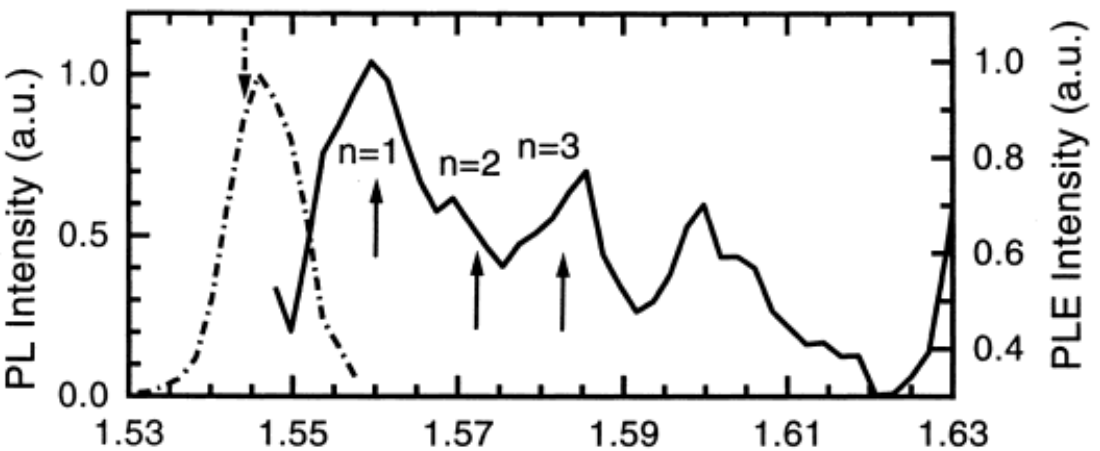

(c)

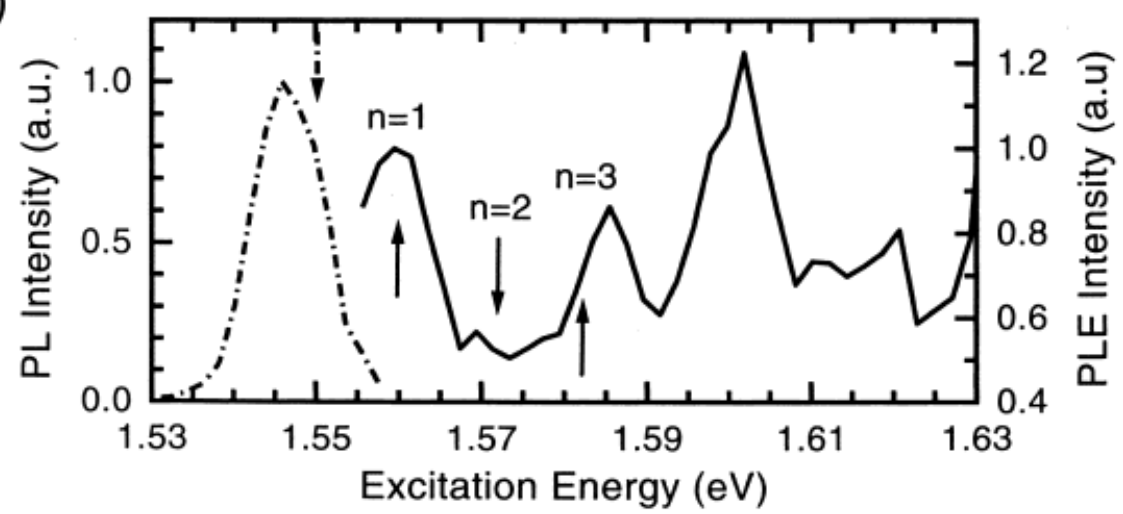

Fig. 2. a) Near-field PLE spectrum recorded for spatially resolved excitation of the QWR sample through the fiber tip at a temperature of $10 \mathrm{~K}$. The intensity of QWR luminescence at $1.544 \mathrm{eV}$ is plotted as a function of excitation energy (abscissa) and of lateral distance between the QWR located at $y=0$ and the fiber tip (ordinate). Only carriers excited at the QWR contribute to the signal. b) Cross-section through a) at $y=0$ (solid line) and QWR near-field luminescence spectrum (dash-dotted line). c) As b), the detection energy being $1.550 \mathrm{eV}$ (a.u. always means arb. units) 
$70 \mathrm{~nm}$ between QWR and sample surface and the finite aperture size of about $200 \mathrm{~nm}$ used in this experiment. A cross-section through this image (Fig. 2b) exhibits several maxima below the onset of QW absorption at $1.63 \mathrm{eV}$. Such peaks are due to the quasione-dimensional subband structure of the QWR and are closely related to the QWR absorption spectrum [18]. Preliminary calculations within the adiabatic approximation [19] suggest that the first peaks are due to the $n=1(1.558 \mathrm{eV}), n=2(1.570 \mathrm{eV})$, and $n=3(1.583 \mathrm{eV})$ heavy-hole to conduction band transitions (arrows in Fig. $2 \mathrm{~b})$. The splitting between the $n=1$ and $n=2$ transitions is on the order of $12 \mathrm{meV}$, in good agreement with the width of this QWR structure of about 40 to $50 \mathrm{~nm}$, as estimated from TEM images and the observed diamagnetic shifts. The strong intensities of the third peak (at $1.583 \mathrm{eV}$ ) and fourth peak (at about $1.60 \mathrm{eV}$ ) suggest a significant contribution from excitonic transitions between valence band states with predominant lighthole character and conduction band states. The role of such transitions has recently been addressed both experimentally [20] and theoretically [21] in investigations of valence band mixing effects on the linear optical properties of quantum wires. Currently polarization resolved experiments are underway that will lead to a definite assignment of these transitions. The small Stokes shift of $13 \mathrm{meV}$ between the first peak and the PL maximum and the narrow PL spectrum (dash-dotted line) indicate the high structural quality of the sample. The small inhomogeneous broadening in the spectra is - most probably - related to exciton localization induced by interface roughness on a length scale between the exciton diameter and the spatial resolution of $300 \mathrm{~nm}$.

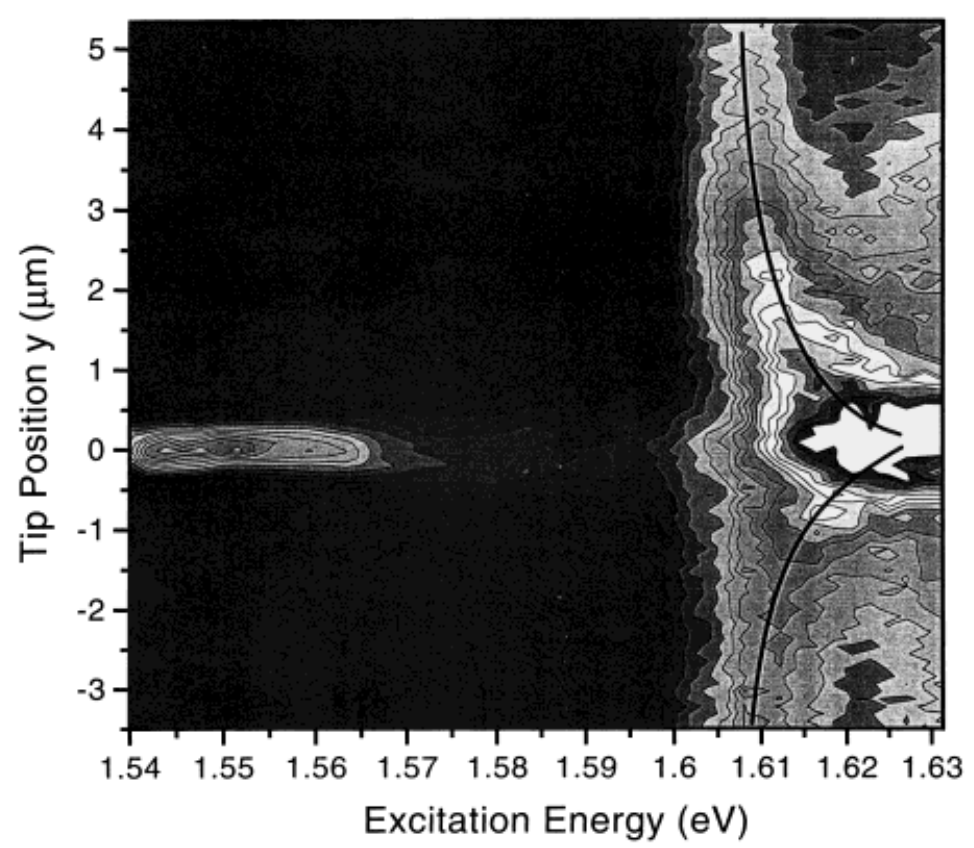

Fig. 3. Near-field PLE spectrum at a sample temperature of $77 \mathrm{~K}$ and a detection energy of $1.533 \mathrm{eV}$, corresponding to the maximum of the QWR emission at this temperature. Notice the pronounced spatial blue-shift of the excitonic QW absorption peak as the excitation tip approaches the wire at $y=0$ 


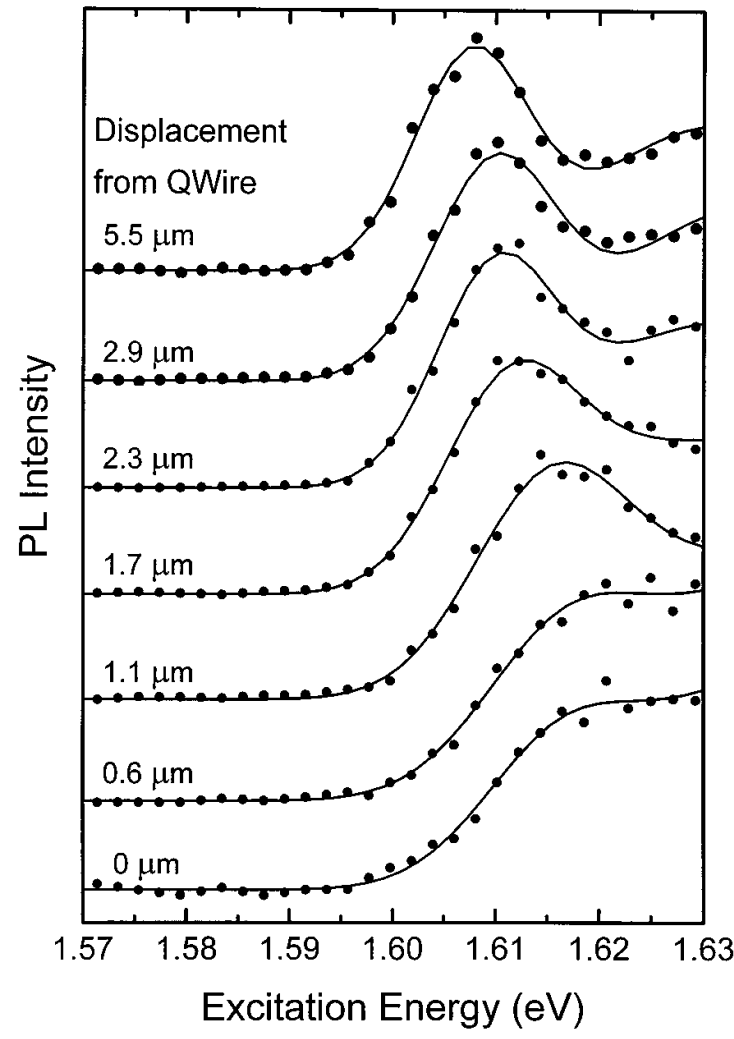

Fig. 4. Cross-sections through Fig. 3. Near-field mesa top QW PLE spectra at fixed excitation positions relative to the QWR location at $y=0$ at a sample temperature of $77 \mathrm{~K}$ and a detection energy of $1.533 \mathrm{eV}$. Experimental data points are shown as solid circles while the solid lines represent fits of the data to Eq. (1)

A similar excitation energy dependence of the QWR luminescence is observed for detection on the high-energy side of the QWR spectrum at $1.550 \mathrm{eV}$ (Fig. 2c). While the energetic position of the peaks is identical to those observed for detection at $1.544 \mathrm{eV}$, their spectral envelope is slightly narrower and the peak intensities differ from those in Fig. 2b. This points to a predominantly inhomogeneously broadened QWR PL spectrum and to exciton localization along the

wire direction. The very intense peak at energies above $1.63 \mathrm{eV}$ is due to the onset of QW absorption. From its separation to the QWR first peak one derives a confinement energy of $80 \mathrm{meV}$. Unlike for QWR absorption, the spatial dependence of the QW absorption peak is not limited by the spatial resolution of our experiment. Its spatial intensity variation is reasonably described by a Gaussian profile with a width of about $850 \mathrm{~nm}$. This width is mainly given by the confinement in the vicinity of the QWR as will be discussed below.

The spatially resolved PLE spectrum changes drastically as the sample temperature is increased to $77 \mathrm{~K}$. In Fig. 3, the luminescence intensity detected near the maximum of the QWR emission at $1.533 \mathrm{eV}$ is plotted as a function of excitation energy and position as the tip is scanned perpendicular to the QWR. The QWR PLE spectrum appears again as a spatially narrow peak around $y=0$, slightly red-shifted with respect to the $10 \mathrm{~K}$ spectrum. The subband-related modulation of the spectrum is washed out due to increased thermal broadening. More important and in contrast to the low-temperature spectrum, strong QWR luminescence is now detected for QW excitation at energies higher than $1.6 \mathrm{eV}$ and at distances $y$ of several $\mu \mathrm{m}$ away from the QWR location. The spectral shape of the PLE spectrum for $y \neq 0$ changes strongly with excitation position. In particular, the lowest maximum of the PLE spectrum shifts towards higher photon energies as the excitation tip approaches the location of the wire at $y=0$. In Fig. 4, we present cross-sections through the image of Fig. 3 for different separations from the 
QWR on the mesa top $(y>0)$ (symbols). The peak in those spectra which is due to the excitonic enhancement of QW absorption shifts by about $14 \mathrm{meV}$ to higher energies as one moves from a distance of $y=5.5 \mu \mathrm{m}$ to the location of the QWR. On the mesa bottom $(y<0)$ (Fig. 3), this shift is even more pronounced with PLE maxima at $1.610 \mathrm{eV}$ for $y=-3.5 \mu \mathrm{m}$ and at $1.628 \mathrm{eV}$ for $y=-0.4 \mu \mathrm{m}$.

The data of Figs. 3 and 4 demonstrate that carriers locally generated within the QW undergo real-space transfer to the location of the QWR where they are trapped into quasi-one-dimensional QWR states and contribute to the QWR emission. The time scale of real-space transfer is given by the recombination lifetime of carriers within the QW. Time-resolved PL measurements under the same excitation conditions give a lifetime of $1.2 \mathrm{~ns}$ at $77 \mathrm{~K}$. This time scale is much longer than typical thermalization and cooling times of carriers, processes occurring on the femto- and picosecond time scale. As a result, the carriers undergoing real-space transfer form a quasi-equilibrium distribution with a temperature close to lattice temperature.

The PLE spectra recorded on the QW are closely related to the local QW absorption spectrum at the specific excitation position while the overall intensity at this position is a measure of the probability for carrier trapping into the QWR. The results in Fig. 4 reveal (i) a pronounced exciton-like feature in the absorption of the QW, and (ii) a blueshift of this maximum with decreasing separation from the QWR. To analyze this behavior in a more quantitative way, we used the Elliott formula [22] for the absorption spectrum $\alpha(E)$ of a quasi-two-dimensional semiconductor,

$$
\alpha(E) \propto \frac{E}{E_{0}}\left(\sum_{n=0}^{\infty} \frac{4}{(n+1 / 2)^{3}} \delta\left(\Delta+\frac{1}{(n+1 / 2)^{3}}\right)+\theta(\Delta) \frac{\exp (\pi / \sqrt{\Delta})}{\cosh (\pi / \sqrt{\Delta})}\right) .
$$

Here, $E$ is the excitation energy, $n$ the quantum number of the bound exciton states, and $E_{0}$ the $2 \mathrm{D}$ exciton binding energy. $\Delta=\left(E-E_{\mathrm{g}}\right) / E_{0}$, with $E_{\mathrm{g}}$ being the bandgap energy. $\theta(\Delta)$ denotes the Heavyside step function. The dominant features are the $n=0$ exciton absorption peak and the continuum absorption, i.e. the second term in Eq. (1). At each excitation position, the QW absorption spectrum in Fig. 4 was fitted to Eq. (1) using a Gaussian profile instead of the $\delta$-function to account for the inhomogeneous broadening of the lines which is probably due to interface roughness of the QW. A similar broadening was used for the step-like continuum absorption (second term in Eq. (1)). The solid lines in Fig. 4 represent the calculated spectra which are in good agreement with the experimentral results. The calculation gives the local bandgap energy $E_{\mathrm{g}}(y)$ as a function of excitation position $y .{ }^{2}$ ) A plot of the bandgap energies of the QW versus $y$ is shown in Fig. 5. On each side of the wire, the potential shows two pronounced maxima, separated by approximately $1 \mu \mathrm{m}$. On the mesa bottom $(y<0)$ the bandgap increases by as much as $18 \mathrm{meV}$ over a length scale of about $2 \mu \mathrm{m}$ as approaching the location of the QWR, while on the mesa top $(y>0)$ the bandgap shift is slightly less pronounced and amounts to about $14 \mathrm{meV}$.

The change in bandgap energy is directly related to a change in the average local thickness of the quantum well, that decreases from about $5.6 \mathrm{~nm}$ on the flat-area mesa

\footnotetext{
${ }^{2}$ ) The change in quantum well thickness near the QWR is correlated with an increase in inhomogeneous broadening. This is directly observed as a pronounced increase in the spectral width of the $n=0$ exciton absorption peak.
} 


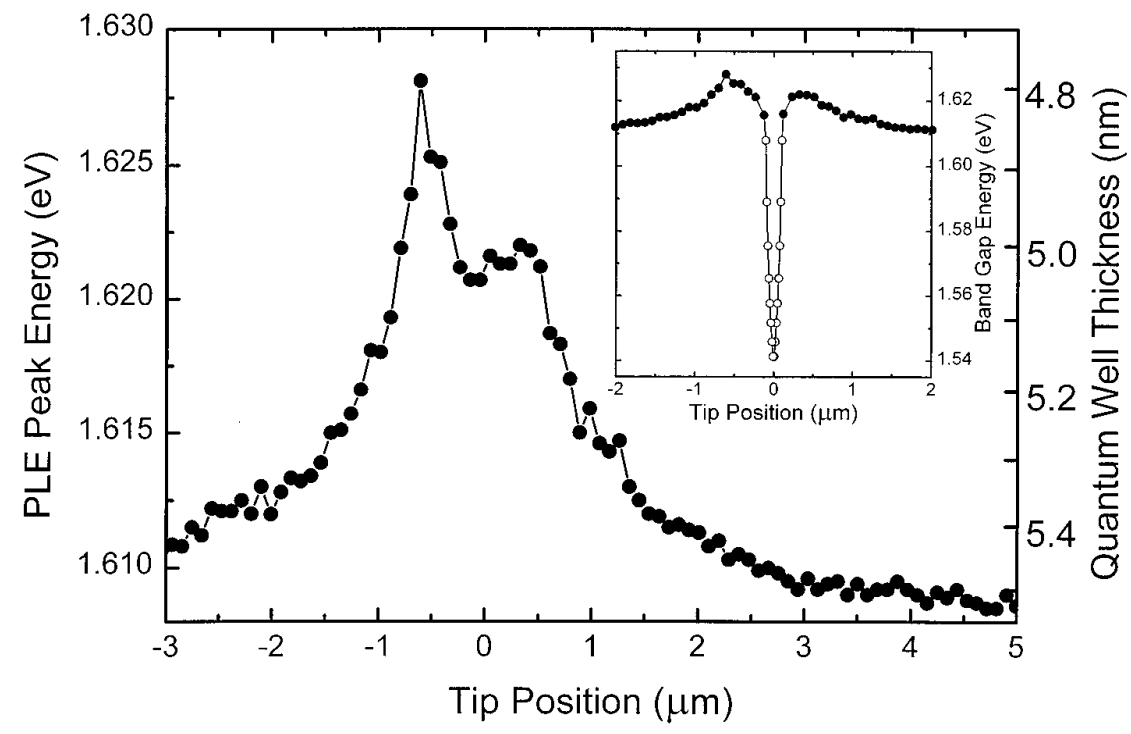

Fig. 5. Lateral band gap profile as obtained from a fit of the QW PLE spectrum shown in Fig. 3 to Eq. (1). The lateral shift in the excitonic QW absorption peak corresponds to a decrease of the average QW thickness in the region close to the QWR. This thinning is a direct consequence of the Ga atom migration towards the sidewall during the growth process and is most pronounced on the mesa bottom

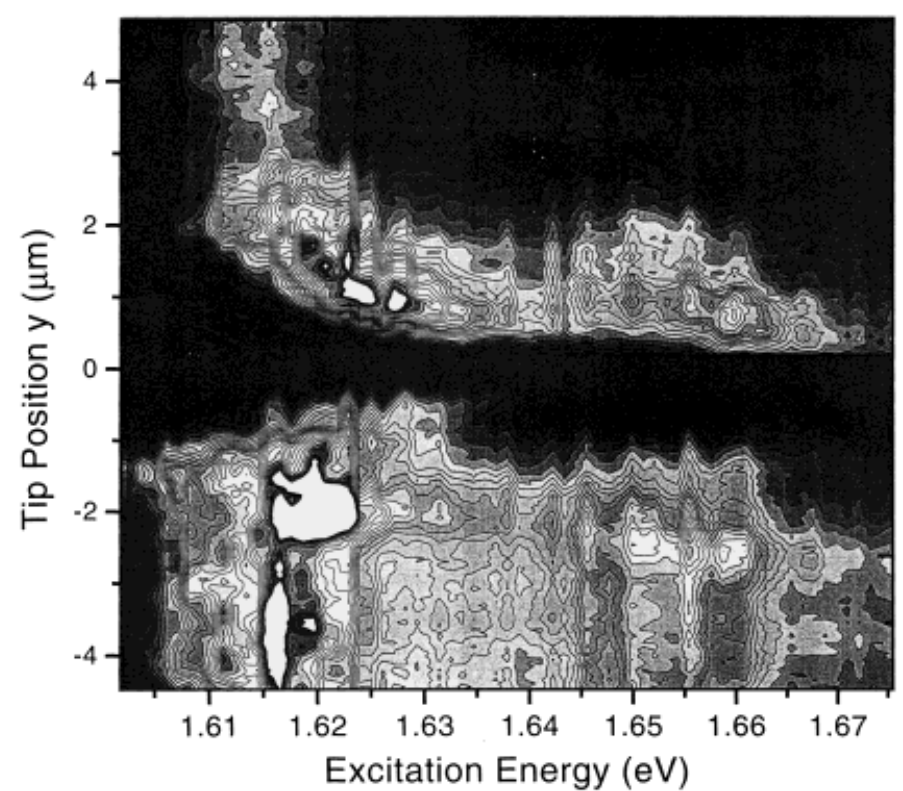

Fig. 6. Near-field PLE spectrum at a sample temperature of $10 \mathrm{~K}$ and a detection energy of $1.598 \mathrm{eV}$, near the maximum of the QW emission at this temperature. For clarity, the signal intensity on the mesa top $(y>0)$ was multiplied by a factor of 2.5. Again the thinning of the QW in the region around the QWR is observed as a blue-shift of the local QW PLE spectrum. The strong spatial and energetic fluctuations of the luminescence intensity are a signature of exciton localization within the QW 
top and bottom down to $4.8 \mathrm{~nm}(y<0)$, respectively $5.0 \mathrm{~nm}(y>0)$, in the vicinity of the wire. This thinning is a consequence of the Ga atom migration towards the sidewall during the growth process and strongly dependent on the specific MBE growth parameters in particular on the substrate temperature. The asymmetry in the bandgap profile on mesa top $(y>0)$ and bottom $(y<0)$ is most likely related to the wet chemical etching procedure that is used to pattern the mesa structure [8], and the resulting changes of Ga migration.

Taking the results of Fig. 5 and the thickness variation of the QW in the central $50 \mathrm{~nm}$ around $y=0$ as determined from cross-sectional TEM images, we derive the complete confinement potential of the quantum well embedded QWR. In the inset of Fig. 5, the bandgap energies are plotted as a function of $y$ for the whole interval between -2 and $+2 \mu \mathrm{m}$. Using this information and a ratio of $2: 1$ for the conduction to valence band offset energy in the $2 \mathrm{D}$ GaAs $/ \mathrm{Al}_{0.5} \mathrm{Ga}_{0.5} \mathrm{As}$ structure, we can now calculate electronic and optical properties of the QWR sample [21]. Preliminary calculations within the adiabatic approximation show that since the height of the local energetic barriers of about $15 \mathrm{meV}$ is only a small fraction of the QWR confinement energy of $80 \mathrm{meV}$, and since the barrier maximum is separated by about $500 \mathrm{~nm}$, their influence on the energies of the lowest QWR states is rather small. Nonetheless these barriers strongly influence the carrier transport within the sample as is discussed below.

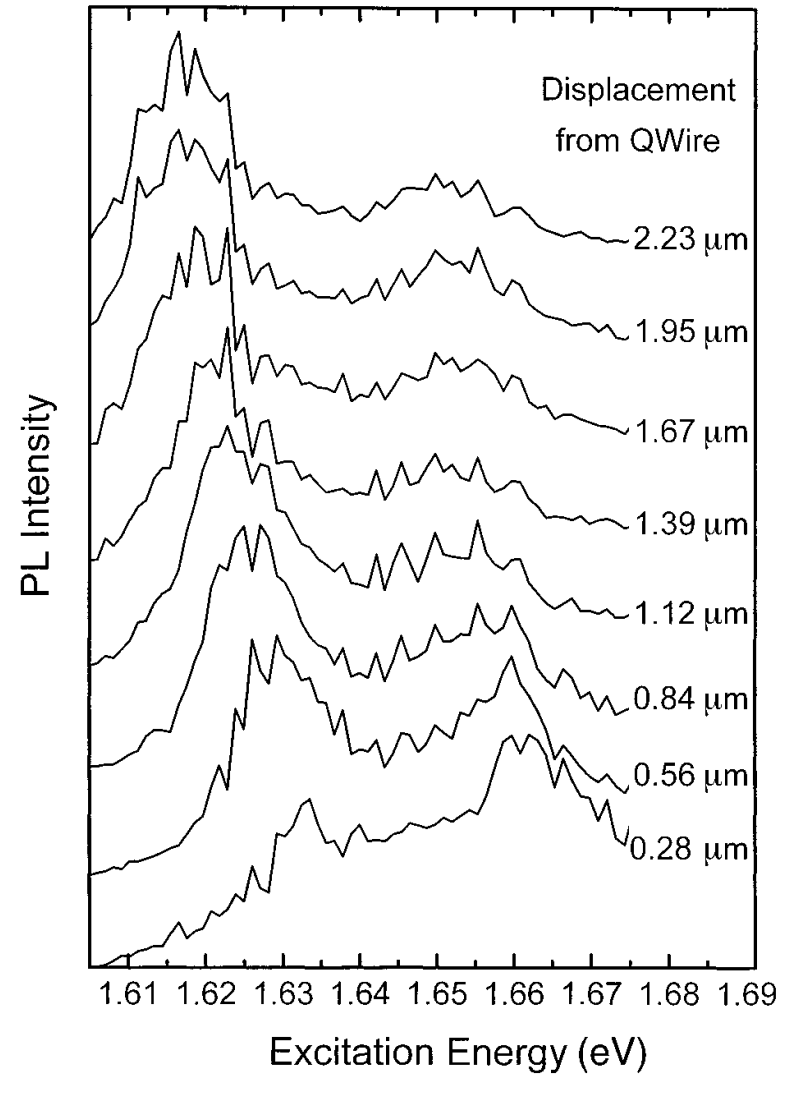

Fig. 7. Cross-sections through Fig. 6. Near-field mesa top QW PLE spectra at fixed excitation positions relative to the QWR location at $y=0$ at a sample temperature of $10 \mathrm{~K}$ and a detection energy of $1.598 \mathrm{eV}$. Notice the strong fluctuations of the luminescence intensity 
To confirm this result, we performed an experiment in which the QW instead of the QWR luminescence was monitored for different separations of the excitation tip from the QWR. At a temperature of $10 \mathrm{~K}$, the QWR PLE spectrum of Fig. 2 shows that carrier trapping into the quantum wire is restricted to a very narrow region around the wire location. Thus, the QW luminescence intensity is less affected by the trapping of carriers into the QWR than at higher temperatures and even more direct information on the QW thickness variations may be obtained. An NSOM PLE spectrum for the detection of the QW luminescence at an energy of $1.598 \mathrm{eV}$ at a sample temperature of $10 \mathrm{~K}$ is shown in Fig. 6. On the mesa top, the excitonic heavy-hole to conduction band absorption appears at about $1.615 \mathrm{eV}$ for $y>2 \mu \mathrm{m}$ and a second light-hole to conduction band transition is centered around $1.65 \mathrm{eV}$. Again the thinning of the QW for excitation near the QWR center is clearly observed as a characteristic blue shift of the first exciton peak in the spectrum (Fig. 7). The analysis of the spectrum gives a similar spatial dependence of the local quantum well thickness as shown in Fig. 5 and thus confirms the interpretation of PLE spectra at $77 \mathrm{~K}$. In contrast to the $77 \mathrm{~K}$ spectrum, the low temperature spectra show pronounced energetic as well as spatial fluctuations of the luminescence intensity. Such fluctuations are a signature of exciton localization within the QW $[23,24]$ due to random monolayer variations of the quantum well width.

\section{Steady-State and Time-Resolved Near-Field Luminescence Spectroscopy: Spatio-Temporal Carrier Transport}

While the presence of the local energetic barriers modifies the energetics of the bound QWR levels only weakly it has a pronounced effect on the carrier transport within the nanostructure. This is demonstrated by using steady-state and time-resolved near-field spectroscopy.

Steady-state near-field PL spectra were recorded at various sample temperatures with spatially resolved excitation at a photon energy of $1.595 \mathrm{eV}$ (Fig. 8). In these experiments, the sample is excited through the NSOM probe and the tip is again scanned along the $y$ direction perpendicular to the QWR located at $y=0$. At a sample temperature of $300 \mathrm{~K}$, QWR emission appears as a peak around $1.47 \mathrm{eV}$ while QW emission is centered around $1.52 \mathrm{eV}$ [25]. The cross-section through the QWR PL spectrum at a detection energy of $1.47 \mathrm{eV}$ shows a narrow peak at $y=0$ arising from direct QWR excitation that is surrounded by broad wings that decay exponentially over a length of several $\mu \mathrm{m}$. This occurrence of QWR luminescence after localized QW excitation involves ambipolar carrier diffusion within the $\mathrm{QW}$ and subsequent trapping into the QWR. A diffusion length of $1.6 \mu \mathrm{m}$ is determined from the exponential PL decay. Knowing the QW lifetime of $2.1 \mathrm{~ns}$ an effective carrier mobility of $488 \mathrm{~cm}^{2} / \mathrm{Vs}$ is obtained [26]. This demonstrates that at room temperature carriers can move across the local barriers because of their large thermal energy of $25 \mathrm{meV}$ and that ambipolar diffusion determines the real-space transfer preceding carrier capture into the QWR.

At low temperatures, a drastically different scenario of carrier transport and trapping is found. At $10 \mathrm{~K}$, QWR emission (around $1.545 \mathrm{eV}$ ) appears as a single narrow peak centered around $y=0$ that is well represented by a Gaussian profile with a width of about $850 \mathrm{~nm}$ [17]. Thus at low temperatures, where the carrier thermal energy is smaller than the barrier height, thermal activation and thus real-space transfer of carriers across 
(a)

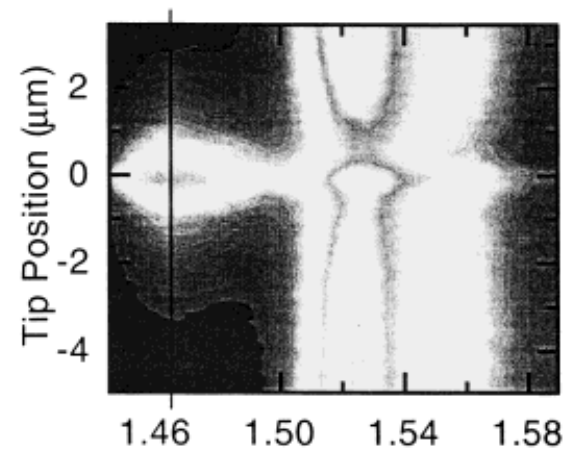

Detection Energy $(\mathrm{eV})$
$\mathrm{T}=293 \mathrm{~K}$

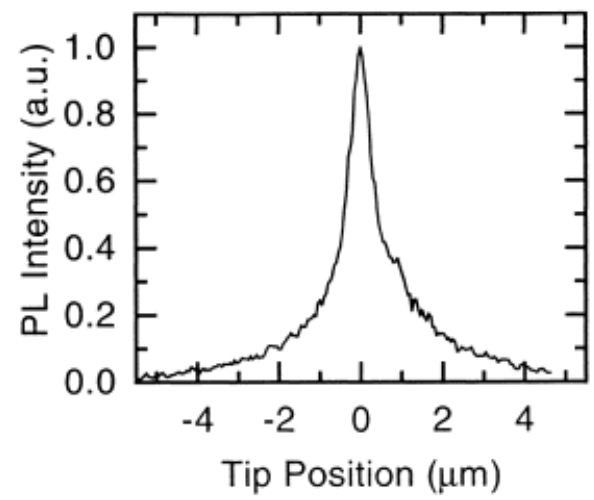

Tip Position $(\mu \mathrm{m})$

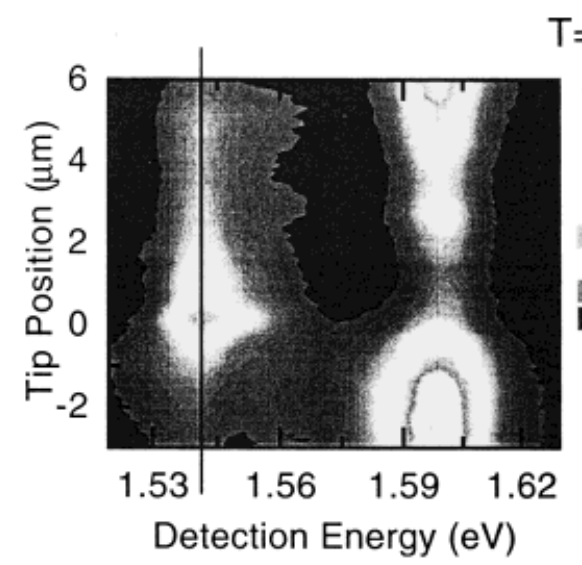

$\mathrm{T}=100 \mathrm{~K}$

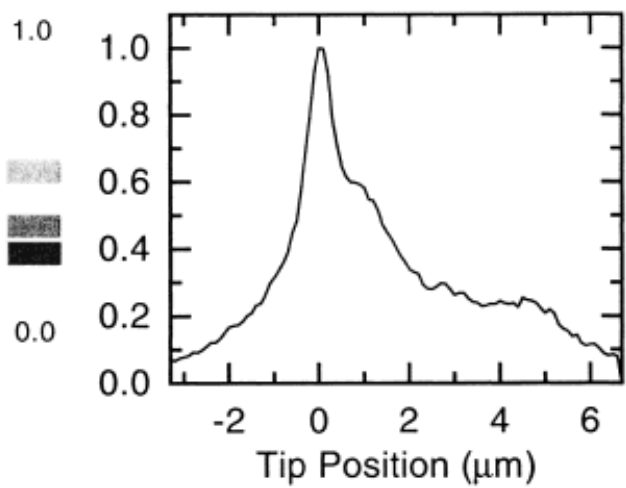

$\mathrm{T}=8 \mathrm{~K}$
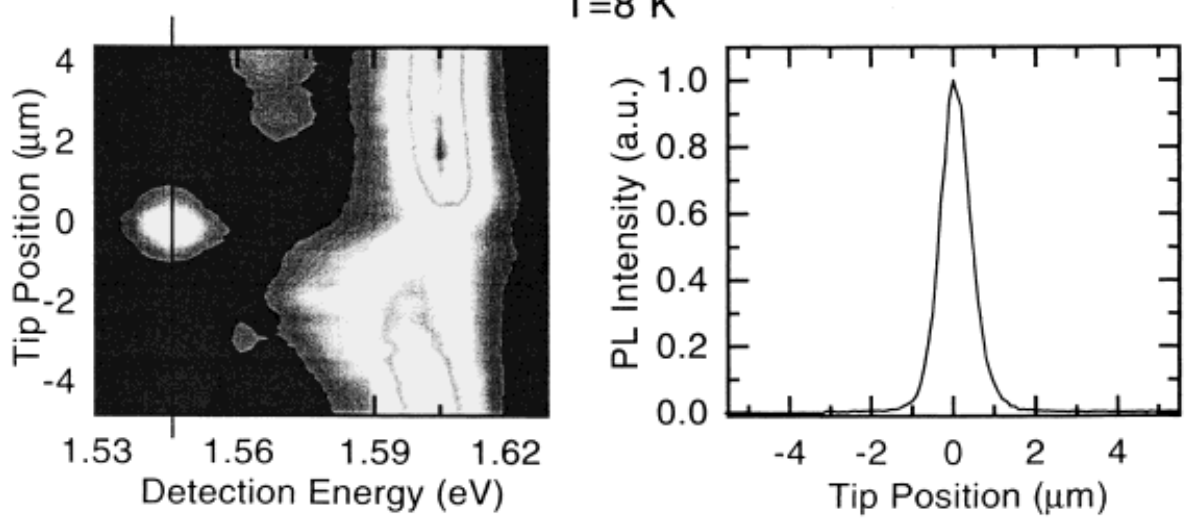

Fig. 8. a) Near-field photoluminescence spectra of the QWR sample for localized excitation at $1.595 \mathrm{eV}$ at three different sample temperatures of 293,100 and $8 \mathrm{~K}$. The luminescence intensity is plotted as a function of detection energy (abscissa) and of lateral distance between the QWR located at $y=0$ and the fiber tip (ordinate). b) Spatial cross-sections through the near-field PL spectra at the energy of the QWR emission maximum 
the barriers are strongly suppressed and trapping of carriers generated within the flat area QW is prohibited. Only generation of carriers within a narrow spatial region between the two energetic barriers separated by about $1 \mu \mathrm{m}$ leads to QWR emission.

At intermediate temperatures around $100 \mathrm{~K}$, the spatial dependence of the QWR luminescence peak around $1.54 \mathrm{eV}$ becomes strongly asymmetric. On the mesa bottom $(y<0$, barrier height $18 \mathrm{meV})$, the QWR luminescence is significantly less intense than on the mesa top $(y>0$, barrier height $14 \mathrm{meV})$. At this temperature, real-space transfer on the mesa top extends over more than $5 \mu \mathrm{m}$ due to the high mobility of holes of about $7000 \mathrm{~cm}^{2} / \mathrm{Vs}$ and the long $2 \mathrm{D}$ carrier lifetime of $1.4 \mathrm{~ns}$. These experiments thus demonstrate directly the pronounced influence of the spatial bandgap variation on the realspace carrier transfer within the $2 \mathrm{D}$ continuum and thus on the carrier trapping dynamics into the $1 \mathrm{D}$ QWR states.

While the steady-state spectra describe the trapping scenario qualitatively, quantitative information can be obtained by combining high spatial and temporal resolution. Since the transport dynamics within the QW occur on the timescale of the $2 \mathrm{D}$ carrier lifetime, i.e. on a nanosecond timescale, first experiments were performed using a timecorrelated single photon counting setup for time-resolved luminescence detection. The sample was locally excited by transmitting picosecond laser pulses through the near-field probe while scanning the probe laterally, perpendicular to the QWR. The time-gated luminescence is then recorded at a fixed detection wavelength as a function the excitation position. Results of such an experiment are presented in Fig. 9 for the detection of QWR emission at $1.544 \mathrm{eV}$ (excitation energy $1.59 \mathrm{eV}$ ) and for a sample temperature of $100 \mathrm{~K}$. For excitation near $y=0$ we find a decay of the wire population with a recombi-

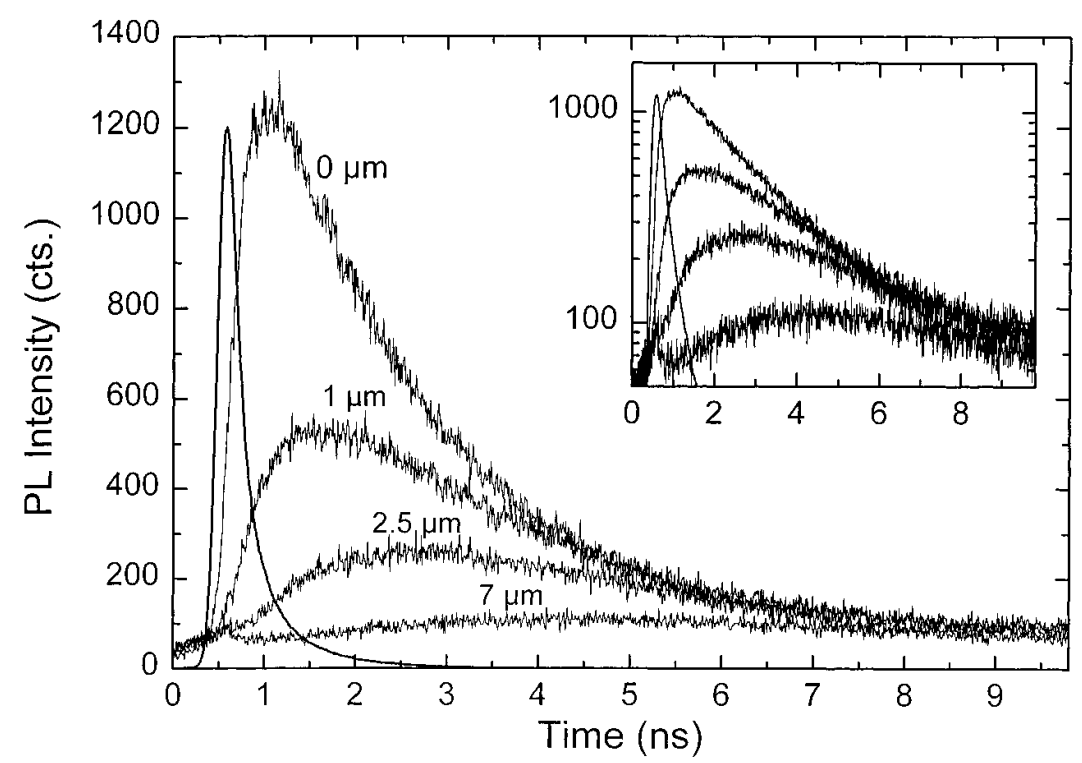

Fig. 9. QWR luminescence decay curves as function of distance between QWR and excitation tip position at a sample temperature of $100 \mathrm{~K}$ and a detection energy of $1.544 \mathrm{eV}$. Excitation occurs on the mesa top $(y>0)$ at $1.59 \mathrm{eV}$. In the inset, the decay curves are shown on a logarithmic scale. Notice the strong temporal shift of the PL peak as a function of excitation position 


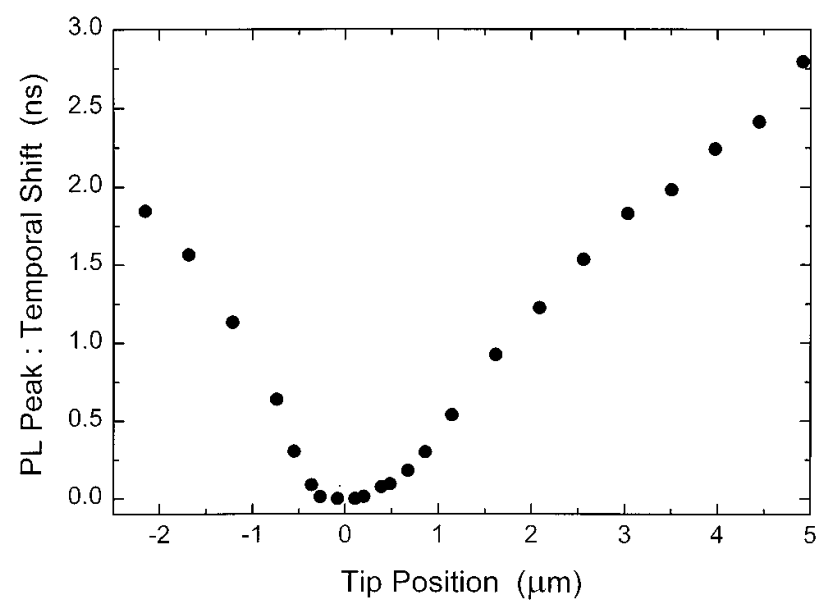

Fig. 10. Temporal shift of the maximum of the QWR PL decay curves in Fig. 9 as a function of excitation position. The shift is indicative of the diffusive transport of carriers between the excitation position and the QWR

nation time of $1.7 \mathrm{~ns}$. As the excitation spot is moved away from the wire position, the appearance of QWR luminescence is temporally delayed, i.e. the maximum of the PL signal shifts to longer times. The shift of the maximum is a clear manifestation of the diffusive real-space transfer of carriers from the excitation position into the quantum wire region. This diffusive real-space transfer precedes carrier trapping into the QWR and energetic relaxation of carriers to the bottom of the QWR potential, processes that are too fast to be resolved with the temporal resolution of this experiment.

In the simplest approximation, one could attempt to describe the carrier diffusion and trapping into the QWR by a single exponential rise of the QWR carrier population, with a rise time that increases with excitation position. Such a model is, however, not in accordance with the experimental data. A quantitative analysis requires a detailed description of the two-dimensional carrier drift-diffusion within the flat parts of the QW and in the barrier regions. Such an analysis is currently underway. At this point, we only present the excitation position dependence of the temporal maximum of the QWR emission (Fig. 10). It is found that the shift of the QWR PL maximum, i.e. the increase of the time interval needed for carrier transport over a specific distance, is more pronounced on the mesa bottom $(y<0)$ than on the mesa top $(y>0)$. This behavior points to a slower carrier transport across the barrier on the mesa bottom which is higher than that on the mesa top (18 versus $14 \mathrm{meV}$, thermal energy of carriers at $100 \mathrm{~K}: 13 \mathrm{meV}$ ). It may also reflect the increased interface roughness on the mesa bottom as a consequence of the wet chemical etching procedure and a resulting loss of carrier mobility.

\section{Summary and Conclusion}

In conclusion, we have demonstrated the potential of low-temperature near-field photoluminescence excitation spectroscopy for locally mapping the bandprofile in semiconductor nanostructures with subwavelength spatial resolution. The confinement potential for a novel quantum-well-embedded GaAs quantum wire structure grown on patterned (311)A GaAs surfaces was extracted using an all-optical technique. The influence of the exact shape of the potential on the carrier transport dynamics within the embedding quantum well and in particular on the trapping of carriers from two-dimensional quan- 
166 Ch. Lienau et al.: Mapping of Local Confinement Potential in Semiconductor Nanostructures

tum well into one-dimensional wire states is revealed in a set of near-field photoluminescence experiments at variable temperatures. By combining high spatial and temporal resolution, the diffusive motion of carriers within the embedding quantum well, carrier trapping and recombination within the quantum wire are monitored directly in both space and time.

\section{References}

[1] J. Christen, F. Bertram, M. Dilger, R. J. Haug, K. Eberl, K. Klitzing, and D. BimBERG, Proc. 23rd Internat. Conf. Physics of Semiconductors, World Scientific Publ. Co., Singapore 1996 (p. 3315).

[2] J. Christen, M. Grundmann, and D. Bimberg, J. Vac. Sci. Technol. B 9, 2358 (1991).

[3] M. E. Rubin, G. Medeiros-Ribeiro, J. J. O’Shea, M. A. Chin, E. Y. Lee, P. M. Petroff, and V. Narayanamurti, Phys. Rev. Lett. 77, 5268 (1996).

[4] C. Eder, J. Smoliner, and G. Strasser, Appl. Phys. Lett. 68, 2876 (1996).

[5] D.W. Pohl, W. Denk, and M. Lanz, Appl. Phys. Lett. 44, 651 (1984).

[6] E. Betzig and J.K. Trautmann, Science 257, 189 (1992).

[7] R. Nötzel, M. Ramsteiner, J. Menniger, A. Trampert, H.-P. Schönherr, L. DÄweritz, and K. H. Ploog, Jpn. J. Appl. Phys. 35, L297 (1996).

[8] R. Nötzel, J. Menniger, M. Ramsteiner, A. Ruiz, H.-P. Schönherr, and K. H. Ploog, Appl. Phys. Lett. 68, 1132 (1996).

[9] R. Nötzel, M. Ramsteiner, J. Menniger, A. Trampert, H.-P. Schönherr, L. Däweritz, and K. H. Ploog, Appl. Phys. Lett. 68, 1132 (1996).

[10] G. Behme, A. Richter, M. Süptitz, and C. Lienau, Rev. Sci. Instrum. 68, 3458 (1997).

[11] E. Betzig, J. K. Trautman, T. D. Harris, J. S. Weiner, and R. L. Kostelak, Science 251, 1468 (1991).

[12] K. Karrai and R. D. Grober, Appl. Phys. Lett. 66, 1842 (1995).

[13] E. Betzig, P. L. Finn, and J. S. Weiner, Appl. Phys. Lett. 60, 2484 (1992).

[14] P. C. Yang, Y. Chen, and M. Vaez-Irvani, J. Appl. Phys. 71, 2499 (1992).

[15] S. Madsen, N. C. R. Jolme, P. S. Ramanujam, S. Hvilsted, J. M. Hvam, and S. Smith, to be published.

[16] G. A. Valoskovic, M. Holton, and G. H. Morrison, Appl. Optics 34, 1215 (1995).

[17] A. Richter, M. Süptitz, Ch. Lienau, T. Elsaesser, M. Ramsteiner, R. Nötzel, and K. H. Ploog, Surface and Interface Anal. 25, 583 (1997).

[18] F. Rossi and E. Molinari, Phys. Rev. Lett. 76, 3642 (1996).

[19] E. Kapon, D. M. Hwang, and R. Bhat, Phys. Rev. Lett. 63, 430 (1989).

[20] F. Vouilloz, D. Y. Oberli, M.-A. Dupertuis, A. Gustaffrson, F. Reinhardt, and E. KAPON, Phys. Rev. Lett. 78, 1580 (1997).

[21] G. Goldoni, F. Rossi, E. Molinari, and A. Fasolino, Phys. Rev. B. 55, 7110 (1997).

[22] H. Haug and S. W. Koch, Quantum Theory of the Optical and Electronic Properties of Semiconductors, World Scientific Publ. Co., Singapore 1997.

[23] D. Gammon, E. S. Snow, B. V. Shanabrook, D. S. Katzer, and D. Park, Science 273, 87 (1996).

[24] H. F. Hess, E. Betzig, T. D. Harris, L. N. Pfeiffer, and K. W. West, Science 264, $1740(1994)$

[25] A. Richter, M. Süptitz, Ch. Lienau, T. Elsaesser, M. Ramsteiner, R. Nötzel, and K. H. PloOG, Ultramicroscopy, in press.

[26] A. Richter, G. Behme, M. Süptitz, Ch. Lienau, T. Elsaesser, R. Nötzel, M. RamSteiner, and K.H. Ploog, Phys. Rev. Lett. 79, 2145 (1997). 